\title{
RRM domain of ALS/FTD-causing FUS interacts with membrane: an anchor of membraneless organelles to membranes?
}

\author{
Yimei Lu, Liangzhong Lim and Jianxing Song* \\ Department of Biological Sciences, Faculty of Science, National University of Singapore; 10 Kent \\ Ridge Crescent, Singapore 119260;
}

Short title: FUS-RRM interacts with membrane

*dbssjx@nus.edu.sg.

The authors have declared that no competing interests exist.

Key words: ALS; FTD; FUS; Low complexity (LC) domain; Membraneless organelle; RNArecognition motif (RRM); Membrane; NMR spectroscopy. 


\begin{abstract}
526-residue FUS functions to self-assemble into reversible droplets/hydrogels, which could be further solidified into pathological fibrils. FUS is composed of $\mathrm{N}$ terminal low-sequence complexity (LC); RNA-recognition motif (RRM) and C-terminal LC domains. FUS belongs to an emerging category of proteins which are capable of forming membraneless organelles in cells via phase separation. On the other hand, eukaryotic cells contain a large network of internal membrane systems. Therefore, it is of fundamental importance to address whether membraneless organelles can interact with membranes. Here we attempted to explore this by NMR HSQC titrations of three FUS domains with gradual addition of DMPC/DHPC bicelle, which mimics the bilayer membrane. We found that both $\mathrm{N}$ - and $\mathrm{C}$-terminal $\mathrm{LC}$ domains showed no significant interaction with bicelle, but its well-folded RRM domain does dynamically interact with bicelle with an interface opposite to that for binding nucleic acids including RNA and ssDNA. If this in vitro observation also occurs in cells, to interact with membrane might represent a mechanism for dynamically organizing membraneless organelles to membranes to facilitate their physiological functions.
\end{abstract}


Fused in Sarcoma/Translocated in Sarcoma (FUS) is encoded by a gene which was first identified as a fusion oncogene in human liposarcomas ${ }^{1,2}$. The 526-residue FUS protein belongs to the FET protein family, which also includes Ewing RNA binding protein (EWS), and TATA-binding protein associated factor (encoded by TAF15) ${ }^{3,4}$. Increasing evidence suggests that FUS is involved in various cellular processes, including cell proliferation, DNA repair, transcription regulation, and multiple levels of RNA and microRNA processing ${ }^{5-7}$. On the other hand, FUS is extensively involved in the pathology of neurodegenerative diseases, particularly in amyotrophic lateral sclerosis (ALS), frontotemporal dementia (FTD) ${ }^{3-15}$. These results suggest that FUS might have a general role in neurodegenerative diseases.

FUS is intrinsically prone to aggregation ${ }^{5-10}$, which is composed of an N-terminal low-sequence complexity (LC) domain (1-267); an RNA-recognition motif (RRM: 285-371) capable of binding a large array of RNA and DNA ${ }^{1,16-19}$; and C-terminal LC domain (371526) (Fig. 1A). RRM is one of the most abundant protein domains in eukaryotes, carrying the conserved RNP1 and RNP2 sequence stretches ${ }^{18}$. Previously the RRM domain of FUS has been determined by NMR spectroscopy to adopt the same overall fold as other RRMs, which consists of a four-stranded $\beta$-sheet and two perpendicular $\alpha$-helices. Very amazingly, although no ALS-causing mutation was identified within RRM, in vivo studies revealed that RRM is required for manifesting FUS cytotoxicity ${ }^{20}$.

Previously, as facilitated by our discovery that unlike well-folded proteins following the "Salting-in" rule that protein solubility increases upon adding salts over the range of low salt concentrations (usually < 300-500 mM), "insoluble" proteins could only be solubilized in aqueous solution with minimized salt concentrations ${ }^{21-23}$, we have successfully studied the ALS-causing and aggregation-prone TDP-43 N-terminal and C-terminal prion-like domains $^{24,25}$. The results decoded that there exists a membrane-interacting subdomain flanked by two prion-like sequences within the TDP-43 C-terminal domain (CTD) ${ }^{25}$. Interestingly, 
we found that ALS-causing genetic, pathological or environmental factors act to eliminate the native folds of human VAPB-MSP domain and superoxide dismutase 1 (SOD1) and consequently the mutants become predominantly disordered in solution without any stable secondary and tertiary structures, which thus have their intrinsic hydrophobic/amphiphilic regions unavoidably exposed to bulk solvent ${ }^{26-30}$. Therefore, these disordered mutants are only soluble in unsalted water but got aggregated in high salt solutions such as in cells with $\sim 150 \mathrm{mM}$ salts. Most unexpectedly, they acquired novel capacity in interacting with membranes energetically driven by forming helices in membrane environments ${ }^{21-23,26-30}$.

Here, we attempted to explore whether FUS domains are able to interact with membranes by monitoring the peak shift of their HSQC peaks upon gradual addition of DMPC/DHPC bicelle, which mimics the bilayer membrane (Fig. 1B). Interestingly, we found that FUS LC domains showed no significant interaction with DMPC/DHPC bicelle, but its well-folded RRM does dynamically interact with bicelle with an interface opposite to that used for binding nucleic acids including RNA and ssDNA as previously mapped out ${ }^{17}$. 


\section{Results}

We first cloned and expressed three FUS domains: namely the N-terminal LC (1-267), RRM (282-371) and C-terminal LC (371-526) domains. While the two LC domains were all found in inclusion body and thus purified under denaturing conditions; the FUS-RRM domain is highly soluble in supernatant and purified under native conditions. Subsequently, we acquired series of HSQC spectra of the ${ }^{15} \mathrm{~N}$-labeled FUS domains in the presence of DMPC/DHPC bicelle (Fig. 1B) at different ratios. The N- and C-terminal LC domains of FUS showed no significant shift of their HSQC peaks even in the presence of bicelle at a ratio up to 1:800 (FUS domain:bicelle), implying that they have no significant ability to interact with membranes.

A
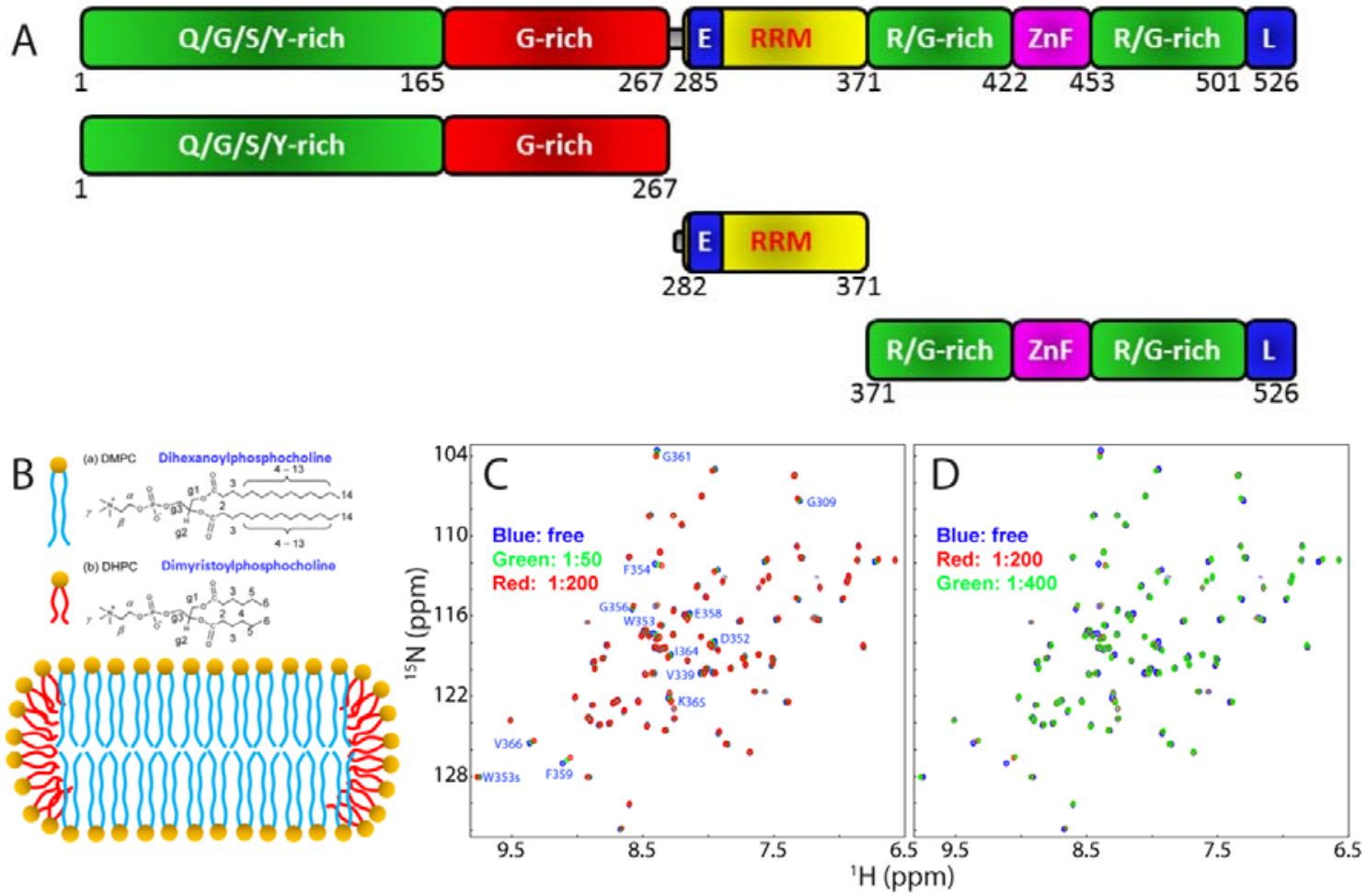

Fig. 1. FUS-RRM interacts with membrane.

(A) Domain organization of the 526-residue FUS protein; and its three dissected domains. (B) Chemical structures of DMPC and DHPC; as well as bicelle structure formed by DMPC/DHPC. (C)-(D) Superimposition of the two-dimensional NMR ${ }^{1} \mathrm{H}_{-}-{ }^{15} \mathrm{~N}$ HSQC spectra of the ${ }^{15} \mathrm{~N}$-labeled FUS-RRM at a concentration of $100 \mu \mathrm{M}$ in $10 \mathrm{mM}$ sodium phosphate at $\mathrm{pH} 6.8$ in the absence (blue), and in the presence of bicelle at different ratios. 
Unexpectedly, however, upon stepwise addition of bicelle, a subset of HSQC peaks of the RRM domain underwent gradual shifts (Fig. 1C). Furthermore, the shifts were mostly saturated at ratio of 1:200 (Fig. 1D). In order to identify the FUS residues with shifted HSQC peaks, we achieved the sequential assignment of our RRM (282-371) by analyzing a pair of triple resonance spectra $\mathrm{HN}(\mathrm{CO}) \mathrm{CACB}$ and $\mathrm{CBCA}(\mathrm{CO}) \mathrm{NH}$ collected on a ${ }^{15} \mathrm{~N}-/^{13} \mathrm{C}$-double labeled sample. Fig. 2A presents the $(\Delta \mathrm{C} \alpha-\Delta \mathrm{C} \beta)$ chemical shifts, which represent a sensitive indicator of the secondary structures of proteins ${ }^{31}$. In addition to the N-terminal residue Ser282, as well as Pro320, Pro344, Pro345 and Pro363, only Lys315, Glu336, Asp343 and Ser360 were not assigned due to overlap or undetectable resonance signals. Most $(\Delta \mathrm{C} \alpha-\Delta \mathrm{C} \beta)$ chemical shifts of our FUS RRM construct are almost the same as those associated with the NMR structure of FUS-RRM (BMRB ID of 17635) over the identical region ${ }^{17}$. 

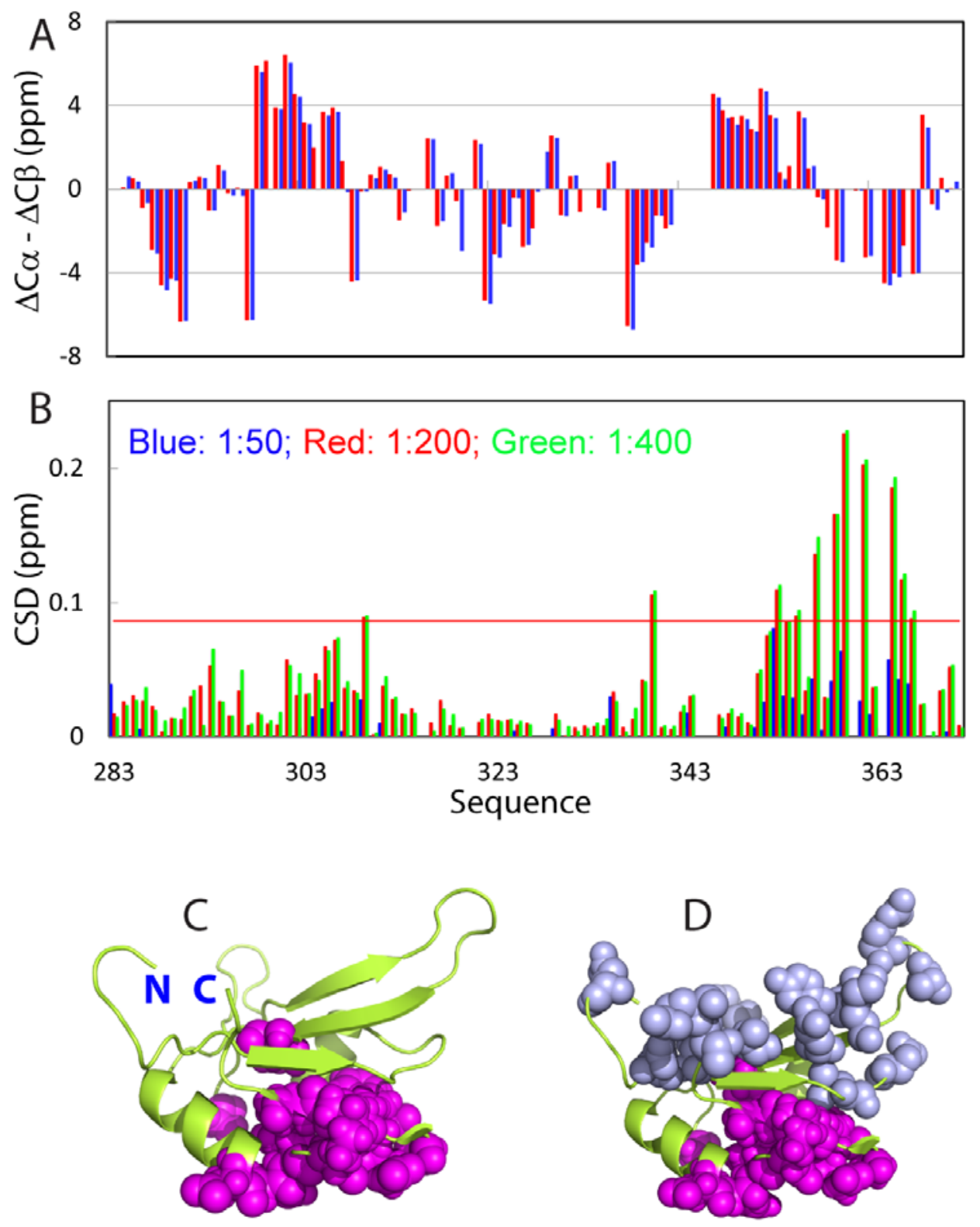

Fig. 2. Interface of FUS-RRM for interacting with membrane.

(A) Residue specific $(\Delta \mathrm{C} \alpha-\Delta \mathrm{C} \beta)$ chemical shifts of the FUS RRM domain collected at $25{ }^{\circ} \mathrm{C}$ in $10 \mathrm{mM}$ phosphate buffer at $\mathrm{pH} 6.8$ (blue) and those previously deposited in BMRB (ID of 17635) (red). (B) Chemical shift difference (CSD) of FUS-RRM in the presence of bicelle at different ratios (RRM:bicelle): 1:50 (blue); 1:200 (red) and 1:400 (green). (C) NMR structure of FUS-RRM (PDB ID of 2LCW) with the residues having significant shifts of HSQC peaks (> average + STDEV [0.086 ppm]) displayed in purple spheres. (D) NMR structure of FUSRRM (PDB ID of 2LCW) further with residues involved in binding ssDNA previously reported ${ }^{17}$ displayed in light blue spheres.

Fig. 2B shows the residue-specific chemical shift difference (CSD) of FUS-RRM in the presence of bicelle at different ratios. Interestingly, upon mapping back the residues with 
significant HSQC peak shifts to the NMR structure, an interesting picture emerges (Fig. 2C): these residues are clustered together to form an interface located on one side of the structure.

We also titrated our FUS-RRM domain with a single-strand DNA (ssDNA) previously used; and found that the residues with significant shifts upon adding this ssDNA are identical to those previously mapped out ${ }^{17}$. Strikingly, the set of residues involved in binding ssDNA have no overlap with the residues whose HSQC peaks significantly shifted upon adding bicelle. The residues critical for binding nucleic acids ${ }^{17}$ are located on the opposite side of the FUS-RRM structure (Fig. 2D). 


\section{Discussion}

Recently, it has been deciphered that proteins containing the LC domains such as prion-like sequences are significantly over-represented by RNA-/DNA binding proteins as exemplified by FUS and TDP-43 $3^{32-44}$. Strikingly, these proteins have been increasingly characterized to be involved in various diseases, particularly neurodegenerative diseases. Most remarkably, their prion-like domains have unique capacity in self-assembling to achieve phase separation which can further transform into fibrils with cross- $\beta$ structures. While it remains highly controversial what are the roles of the diverse structures formed during selfassembly in physiology and pathology, it becomes clear that the phase separation represents a key mechanism to form membraneless compartments or organelles in cells ${ }^{32-51}$.

On the other hand, eukaryotic cells contain a large network of internal membrane systems. This inspires us to hypothesize that the cellular functions of some membraneless organelles formed by phase separation might be facilitated if they can have interactions with membranes to different degrees. For example, the localization to membrane might increase the local concentration of these proteins to enhance self-assembly. Indeed, previously, we found the presence of a hydrophobic subdomain within the TDP-43 CTD, which can transform from partially folded state into a well-folded $\Omega$-loop-helix motif upon interacting with membranes ${ }^{25}$. Most importantly, we showed that the self-assembly was significantly enhanced if the TDP-43 CTD was anchored into bicelle ${ }^{25}$.

Here we found the well-folded FUS-RRM domain also interacts with bicelle by using a small set of residues. Previously, we have investigated but found that the wild-type VAPBMSP domain and the matured SOD1 showed no detectable interaction with bicelle and even DPC-micelle ${ }^{26-28}$. Moreover, the interfaces of FUS-RRM for interacting with nucleic acids and membrane are located on opposite sides of the structures. This strongly implies that our current in vitro observation that FUS-RRM is capable of interacting with membrane might 
bioRxiv preprint doi: https://doi.org/10.1101/122671; this version posted March 31, 2017. The copyright holder for this preprint (which was not certified by peer review) is the author/funder. All rights reserved. No reuse allowed without permission.

indeed occur in cells. Nevertheless, this observation certainly needs in vivo confirmation although it might be extremely challenging due to its dynamic nature. 


\section{Methods}

\section{Preparation of recombinant proteins.}

The DNA fragments encoding three dissected domains of FUS (Fig. 1A) were amplified by PCR reactions from FUS cDNA and subsequently cloned into a modified vector pET28a as we previously used for the TDP-43 prion-like domain ${ }^{25}$. The expression vectors were subsequently transformed into and overexpressed in Escherichia coli BL21 (DE3) cells (Novagen). The recombinant proteins of FUS (1-267) and FUS (371-526) were found only in inclusion body while RRM was highly soluble in supernatant. As a result, for FUS (1-267) and FUS (371-526), the pellets were first dissolved in a phosphate buffer ( $\mathrm{pH}$ 8.5) containing $8 \mathrm{M}$ urea and subsequently purified by a $\mathrm{Ni}^{2+}$-affinity column (Novagen) under denaturing conditions in the presence of $8 \mathrm{M}$ urea. The fractions containing the recombinant proteins were acidified by adding $10 \%$ acetic acid and subsequently purified by reverse-phase (RP) HPLC on a C4 column eluted by water-acetonitrile solvent system. The HPLC elution containing pure recombinant proteins were lyophilized. For RRM, the recombinant proteins were purified by a $\mathrm{Ni}^{2+}$-affinity column (Novagen) under native condition, followed by a further purification by FPLC on a gel-filtration column.

The generation of the isotope-labelled proteins for NMR studies followed a similar procedure except that the bacteria were grown in M9 medium with the addition of $\left({ }^{15} \mathrm{NH}_{4}\right)_{2} \mathrm{SO}_{4}$ for ${ }^{15} \mathrm{~N}$ labeling and $\left({ }^{15} \mathrm{NH}_{4}\right)_{2} \mathrm{SO}_{4} /\left[{ }^{13} \mathrm{C}\right]$-glucose for double labelling ${ }^{24,25}$. The purity of the recombinant proteins was checked by SDS-PAGE gels and their molecular weights were verified by a Voyager STR matrix-assisted laser desorption ionization time-offlight-mass spectrometer (Applied Biosystems). The concentration of protein samples was determined by the UV spectroscopic method in the presence of $8 \mathrm{M}$ urea. Briefly, under the 
denaturing condition, the extinct coefficient at $280 \mathrm{~nm}$ of a protein can be calculated by adding up the contribution of Trp, Tyr and Cys residues ${ }^{56}$.

\section{NMR experiments}

All NMR experiments were acquired on an $800 \mathrm{MHz}$ Bruker Avance spectrometer equipped with pulse field gradient units as described previously ${ }^{24,25}$. For characterizing the solution structure of the FUS RRM domain, a pair of triple-resonance experiments HNCACB, $\mathrm{CBCA}(\mathrm{CO}) \mathrm{NH}$ were collected for the sequential assignment on a ${ }^{15} \mathrm{~N}-/^{13} \mathrm{C}$-double labelled sample of $400 \mu \mathrm{M}$.

Here a membrane-mimetic system DMPC/DHPC bicelle was used to assess the membrane-interaction of FUS-RRM. The large bicelle to better mimic bilayer membrane was prepared by mixing up DMPC and DHPC at a q value of 4 as previously described ${ }^{25,53}$. At this ratio, the disk-shaped bicelle with a diameter of $\sim 460 \AA$ is formed in which DMPC constitutes a bilayered section surrounded by a rim of DHPC (Fig. 1B). The FUS-RRM sample for titrating with bicelle was prepared at $100 \mu \mathrm{M}$ in $10 \mathrm{mM}$ sodium phosphate buffer at $\mathrm{pH} 6.8$. 


\section{References}

1. Crozat A, Aman P, Mandahl N, Ron D. Fusion of CHOP to a novel RNA-binding protein in human myxoid liposarcoma. Nature. 363, 640-644 (1993).

2. Rabbitts TH, Forster A, Larson R, Nathan P. Fusion of the dominant negative transcription regulator CHOP with a novel gene FUS by translocation $\mathrm{t}(12 ; 16)$ in malignant liposarcoma. Nat. Genet. 4, 175-180 (1993).

3. Tan AY, Manley JL. The TET family of proteins: functions and roles in disease. J. Mol. Cell Biol. 1, 82-92 (2009).

4. Schwartz JC, Cech TR, Parker RR. Biochemical Properties and Biological Functions of FET Proteins. Annu Rev Biochem. 84, 355-79 (2015).

5. Lagier-Tourenne C, Polymenidou M, Cleveland DW. TDP-43 and FUS/TLS: emerging roles in RNA processing and neurodegeneration. Hum. Mol. Genet. 19, R46-R64 (2010).

6. Ling SC, Polymenidou M, Cleveland DW. Converging mechanisms in ALS and FTD: disrupted RNA and protein homeostasis. Neuron. 79, 416-438 (2013).

7. Deng H, Gao K, Jankovic J. The role of FUS gene variants in neurodegenerative diseases. Nat Rev Neurol. 10, 337-48 (2014).

8. Neumann M, et al. Abundant FUS-immunoreactive pathology in neuronal intermediate filament inclusion disease. Acta Neuropathol. 118, 605-616 (2009).

9. Doi H, Koyano S, Suzuki Y, Nukina N, Kuroiwa Y. The RNA-binding protein FUS/TLS is a common aggregate-interacting protein in polyglutamine diseases. Neurosci. Res. 66, 131$133(2010)$.

10. Woulfe J, Gray DA, Mackenzie IR. FUS-immunoreactive intranuclear inclusions in neurodegenerative disease. Brain Pathol. 20, 589-597 (2010). 
11. Kwiatkowski TJ Jr, et al. Mutations in the FUS/TLS gene on chromosome 16 cause familial amyotrophic lateral sclerosis. Science. 323, 1205-1208 (2009).

12. Vance $\mathrm{C}$, et al. Mutations in FUS, an RNA processing protein, cause familial amyotrophic lateral sclerosis type 6. Science. 323, 1208-1211 (2009).

13. Broustal O, et al. FUS mutations in frontotemporal lobar degeneration with amyotrophic lateral sclerosis. J. Alzheimers Dis. 22, 765-769 (2010).

14. Van Langenhove $\mathrm{T}$, et al. Genetic contribution of FUS to frontotemporal lobar degeneration. Neurology. 74, 366-371 (2010).

15. Merner ND, et al. Exome sequencing identifies FUS mutations as a cause of essential tremor. Am. J. Hum. Genet. 91, 313-319 (2012).

16. Zinszner H, Sok J, Immanuel D, Yin Y, Ron D. TLS (FUS) binds RNA in vivo and engages in nucleo-cytoplasmic shuttling. J. Cell Sci. 110, 1741-1750 (1997).

17. Liu X, et al. The RRM domain of human fused in sarcoma protein reveals a noncanonical nucleic acid binding site. Biochim Biophys Acta. 1832, 375-385 (2013).

18. Cléry A, Blatter M, Allain FH. RNA recognition motifs: boring? Not quite. Curr Opin Struct Biol. 18, 290-298 (2008).

19. Dreyfuss G, Swanson MS, Piñol-Roma S. Heterogeneous nuclear ribonucleoprotein particles and the pathway of mRNA formation. Trends Biochem Sci. 13, 86-91 (1988).

20. Sun Z, et al. Molecular determinants and genetic modifiers of aggregation and toxicity for the ALS disease protein FUS/TLS. PLoS Biol. 9, e1000614 (2011).

21. Song J. Insight into "insoluble proteins" with pure water. FEBS Lett. 583, 953-959 (2009).

22. Song J. Why do proteins aggregate? "Intrinsically insoluble proteins" and "dark mediators" revealed by studies on "insoluble proteins" solubilized in pure water. F1000 Res. 2, 94 (2013). 
23. Song J. Transforming cytosolic proteins into "insoluble" and membrane-toxic forms triggering diseases/aging by genetic, pathological or environmental factors. Protein Pept. Lett. DOI: $10.2174 / 0929866524666170209154001$ (2017).

24. Qin H, Lim L, Wei Y, Song J. TDP-43 N terminus encodes a novel ubiquitin-like fold and its unfolded form in equilibrium that can be shifted by binding to ssDNA. Proc Natl Acad Sci U S A. 111, 18619-18624 (2014).

25. Lim L, Wei Y, Lu Y, Song J. ALS Causing Mutations Significantly Perturb the SelfAssembly and Interaction with Nucleic Acid of the Intrinsically Disordered Prion-Like Domain of TDP-43. PLoS Biol. 14, e1002338 (2016).

26. Shi J, Lua S, Tong JS, Song J. Elimination of the native structure and solubility of the hVAPB MSP domain by the Pro56Ser mutation that causes amyotrophic lateral sclerosis. Biochemistry. 49, 3887-397 (2010).

27. Qin H, Wang W, Song J. ALS-causing P56S mutation and splicing variation on the hVAPB MSP domain transform its $\beta$-sandwich fold into lipid-interacting helical conformations. Biochem Biophys Res Commun. 431, 398-403 (2013).

28. Qin H, Lim L, Wei Y, Gupta G, Song J. Resolving the paradox for protein aggregation diseases: NMR structure and dynamics of the membrane-embedded P56S-MSP causing ALS imply a common mechanism for aggregation-prone proteins to attack membranes. F1000Res. 2, 221 (2013).

29. Lim L, Lee X, Song J. Mechanism for transforming cytosolic SOD1 into integral membrane proteins of organelles by ALS-causing mutations. Biochim Biophys Acta. 1848, 17 (2015).

30. Lim L, Song J. SALS-linked WT-SOD1 adopts a highly similar helical conformation as FALS-causing L126Z-SOD1 in a membrane environment. Biochim Biophys Acta. 1858, 2223-2230 (2016). 
31. Dyson HJ, Wright PE. Unfolded proteins and protein folding studied by NMR. Chem. Rev. 104, 3607-3622 (2004).

32. Michelitsch MD, Weissman JS. A census of glutamine/asparagine-rich regions: implications for their conserved function and the prediction of novel prions. Proc. Natl. Acad. Sci. USA. 97, 11910-11915 (2000).

33. Kato M, et al. Cell-free formation of RNA granules: low complexity sequence domains form dynamic fibers within hydrogels. Cell. 149, 753-767 (2012).

34. Han TW, et al. Cell-free formation of RNA granules: bound RNAs identify features and components of cellular assemblies. Cell. 149, 768-779 (2012).

35. March ZM, King OD, Shorter J. Prion-like domains as epigenetic regulators, scaffolds for subcellular organization, and drivers of neurodegenerative disease. Brain Res. 1647, 9-18 (2016).

36. Alberti S, Halfmann R, King O, Kapila A, Lindquist S. A systematic survey identifies prions and illuminates sequence features of prionogenic proteins. Cell. 137, 146-158 (2009).

37. Li YR, King OD, Shorter J, Gitler AD. Stress granules as crucibles of ALS pathogenesis. J Cell Biol. 201, 361-372 (2013).

38. Schwartz JC, Wang X, Podell ER, Cech TR. RNA seeds higher-order assembly of FUS protein. Cell Rep. 5, 918-925 (2013).

39. Burke KA, Janke AM, Rhine CL, Fawzi NL. Residue-by-Residue View of In Vitro FUS Granules that Bind the C-Terminal Domain of RNA Polymerase II. Mol Cell. 60, 231-241 (2015).

40. Guo L, Shorter J. Biology and Pathobiology of TDP-43 and Emergent Therapeutic Strategies. Cold Spring Harb Perspect Med. DOI:10.1101/cshperspect.a024554. (2016).

41. Patel A, et al. A Liquid-to-Solid Phase Transition of the ALS Protein FUS Accelerated by Disease Mutation. Cell. 162, 1066-1077 (2015). 
42. Murakami T, et al. ALS/FTD Mutation-Induced Phase Transition of FUS Liquid Droplets and Reversible Hydrogels into Irreversible Hydrogels Impairs RNP Granule Function. Neuron. 88, 678-690 (2015).

43. Elbaum-Garfinkle S, Brangwynne CP. Liquids, Fibers, and Gels: The Many Phases of Neurodegeneration. Dev Cell. 35, 531-532 (2015).

44. Toretsky JA, Wright PE. Assemblages: functional units formed by cellular phase separation. J Cell Biol. 206, 579-588 (2014).

45. Wei Y, Lim L, Wang L, Song J. Inter-domain interactions of TDP-43 as decoded by NMR. Biochem Biophys Res Commun. 473, 614-619 (2016).

46. Xiang S, et al. The LC Domain of hnRNPA2 Adopts Similar Conformations in Hydrogel Polymers, Liquid-like Droplets, and Nuclei. Cell. 163, 829-39 (2015).

47. Bowden H, Dormann D. Altered mRNP granule dynamics in FTLD pathogenesis. $J$ Neurochem. 138, 112-133 (2016).

448. Schmidt HB, Rohatgi R. In Vivo Formation of Vacuolated Multi-phase Compartments Lacking Membranes. Cell Rep. 16, 1228-36 (2016).

49. Conicella AE, Zerze GH, Mittal J, Fawzi NL. ALS Mutations Disrupt Phase Separation Mediated by $\alpha$-Helical Structure in the TDP-43 Low-Complexity C-Terminal Domain. Structure. 24, 1537-1549 (2016).

50. Lu Y, Lim L, Tan Y, Wang L, Song J. Mechanisms of self-assembly and fibrillization of the prion-like domains bioRxiv, 065631. doi: https://doi.org/10.1101/065631 (2016).

51. Wei Y, Lim L, Wang L, Song J. ALS-causing cleavages of TDP-43 abolish its RRM2 structure and unlock CTD for enhanced aggregation and toxicity. Biochem Biophys Res Commun. DOI: 10.1016/j.bbrc.2017.02.139 (2017).

52. Pace CN, Vajdos F, Fee L, Grimsley G, Gray T. How to measure and predict the molar absorption coefficient of a protein. Protein Sci. 4, 2411-2423 (1995). 
bioRxiv preprint doi: https://doi.org/10.1101/122671; this version posted March 31, 2017. The copyright holder for this preprint (which was not certified by peer review) is the author/funder. All rights reserved. No reuse allowed without permission.

53. Dürr UH, Gildenberg M, Ramamoorthy A. (2012) The magic of bicelles lights up membrane protein structure. Chem Rev. 112:6054-6074. 


\section{Acknowledgement}

This study is supported by Ministry of Education of Singapore (MOE) Tier 2

MOE2015-T2-1-111 to Jianxing Song. The funders had no role in study design, data collection and analysis, decision to publish, or preparation of the manuscript.

\section{Author Contributions}

Conceived and designed the experiments: JXS; Performed the experiments: YML LZL. Analyzed the data: YML LZL JXS. Prepared figures and wrote the paper: JXS. 